\title{
NAFLD or MAFLD: Which Has Closer Association With All-Cause and Cause-Specific Mortality?-Results From NHANES III
}

OPEN ACCESS

Edited by:

Aldo Torre

Instituto Nacional de Ciencias Médicas y Nutrición Salvador Zubirán (INCMNSZ), Mexico

Reviewed by: Angelo Armandi, University of Turin, Italy Francesco Tovoli, University of Bologna, Italy

*Correspondence: Xiantong Zou

evazou@bjmu.edu.cn Linong Ji

jiln@bjmu.edu.cn

tThese authors have contributed equally to this work

Specialty section: This article was submitted to Gastroenterology, a section of the journal Frontiers in Medicine

Received: 11 April 2021 Accepted: 02 June 2021 Published: 01 July 2021

Citation: Huang Q, Zou X, Wen X, Zhou X and Ji L (2021) NAFLD or MAFLD: Which

Has Closer Association With

All-Cause and Cause-Specific Mortality?-Results From NHANES III. Front. Med. 8:693507.

doi: 10.3389/fmed.2021.693507

\section{Qi Huang ${ }^{\dagger}$, Xiantong Zou ${ }^{\star}$, Xin Wen, Xianghai Zhou and Linong Ji* \\ Department of Endocrinology and Metabolism, Peking University People's Hospital, Beijing, China}

Background: The recent change of terminology from non-alcoholic fatty liver disease (NAFLD) to metabolic dysfunction-associated fatty liver disease (MAFLD) has raised heated discussion. We aim to investigate the association of MAFLD or NAFLD with all-cause and cause-specific mortality to compare the outcomes of the two diagnostic criteria in population-based study.

Methods: We recruited 12,480 participants from the Third National Health and Nutrition Examination Survey (NHANES III) with matched mortality data in 2015. Participants were divided into four groups for survival analysis: without NAFLD or MAFLD, with only NAFLD, only MAFLD. Cox proportional hazard regression was used to estimate multivariable-adjusted hazard ratios (HRs) and 95\% confidence intervals (Cls) for all-cause and cause-specific mortality. Subgroup analysis were applied in MAFLD patients.

Results: The weighted prevalence of MAFLD and NAFLD was relatively 27.4 and 27.9\%. Participants with NAFLD or MAFLD were largely overlapped (weighted Cohen's kappa coefficient 0.76). MAFLD increased the overall risk for total mortality in a greater magnitude than NAFLD [HR 2.07 (95\% Cl 1.86, 2.29) vs. 1.47 (1.20, 1.79)], However, the difference was non-significant after metabolic parameters were adjusted. Risks for cardiovascular, neoplasm, and diabetes-related mortality were similar between MAFLD and NAFLD. Referring to individuals without both NAFLD and MAFLD, individuals with only NAFLD showed reduced total mortality [HR $0.48(0.34,0.68)]$ and neoplasm mortality [HR $0.46(0.24,0.89)]$ in crude. Nevertheless, individuals with only MAFLD independently increased the risk for total mortality [adjusted HR $1.47(1.22,1.77)$ ] and neoplasm mortality [aHR $1.58(1.09,2.28)]$. The risk for overall mortality in MAFLD was consistent between subgroups except for race-ethnicity and whether secondary to viral hepatitis.

Conclusions: Participants with MAFLD or NAFLD were highly concordant. MAFLD showed greater risk for all-cause mortality and equal risk for cause-specific mortality referring to NAFLD. The new terminology excluded participants with lower mortality risk and included participants with higher risk. Drug development for MAFLD should consider ethnic differences.

Keywords: NAFLD, MAFLD, mortality, NHANES, diagnosis 


\section{INTRODUCTION}

Non-alcoholic fatty liver disease (NAFLD) is the most common liver disease affecting around one-quarter of the population worldwide, causing a global economic burden (1). The definition of NAFLD requires presence of fat on imaging to liver biopsy and exclusion of other liver diseases e.g., excess alcohol intake, drug-induced liver injury, and viral hepatitis (2). NAFLD is also regarded as a "metabolic disease" since it is closely associated with metabolic disorders including obesity, dyslipidemia, and diabetes mellitus (3), of which the common etiology is insulin resistance (4). Patients with NAFLD have a higher risk of cardiovascular events. The leading cause of mortality in NAFLD patients is cardiovascular disease and major excess mortality may result from extrahepatic cancer $(1,5,6)$. A meta-analysis suggested that NAFLD was independently associated with increased absolute risk of all-cause mortality, but the risk for cardiovascular and cancer mortality was similar between NAFLD and non-NAFLD participants $(1,6-8)$.

The progression and prognosis of NAFLD are highly heterogeneous. Only $2-3 \%$ of participants progressed from steatosis to non-alcoholic steatohepatitis (NASH) and advanced fibrosis. Liver related mortality only explained $7 \%$ of deaths among NAFLD patients $(9,10)$. At the beginning of 2020, experts from the European Liver Patient's Association (ELPA) proposed a change of nomenclature from NAFLD to metabolic dysfunctionassociated fatty liver disease (MAFLD), which was mainly defined as liver fat deposition along with obesity, diabetes, or combined metabolic disorders $(11,12)$. This change emphasized the importance of metabolic disorder complicated with fatty liver regardless of the heterogeneous etiology since the exclusion of other liver diseases was no longer required.

Intense dispute raised over the change of the terminology since whether the change from NAFLD to MAFLD can benefit clinical practice and drug development is largely unknown. Studies suggested that participants with NAFLD and MAFLD were highly compatible with each other, and patients with MAFLD were more likely to have worse metabolic profiles than NAFLD $(13,14)$. Other experts concerned that the change may exclude patients with worse outcome, such as participants with "lean NAFLD" who have lower BMI and better metabolic profile, and participants with severe hepatic steatosis who may have more liver fibrosis and elevated long-term comorbidities $(13,15)$. In addition, although MAFLD may reflect relevant risk factors as a metabolic disease, whether this change is necessary regarding biomarker identification, treatment strategy and prognosis is largely unknown (16).

A key question to be answered is whether the change from NAFLD to MAFLD could affect the association between fatty liver and clinical outcomes. A study from Japan suggested that individuals with NAFLD and MAFLD had similar metabolic traits at baseline as well as incidence for cardiovascular events after a 7-year follow-up (17). However, the association between MAFLD and mortality in the long run was largely unknown. Here we aimed to use the National Health and Nutrition Examination Survey III cohort and the follow-up mortality data to answer whether the terminology MAFLD is superior to NAFLD regarding their long-term mortality risk and causespecific mortality risk.

\section{MATERIALS AND METHODS}

\section{Study Design and Participants}

The Third National Health and Nutrition Examination Survey (NHANES III) profiles health estimates of civilian noninstitutionalized US population using a multistage, stratified sampling design from 1988 to 1994 (18). Ultrasound grading of hepatic steatosis was combined at baseline. Linked mortality information through December 31, 2015, was provided by the National Center for Health Statistics (NCHS) of the Centers for Disease Control and Prevention (CDC).

In NHANES III, 14,797 participants aged 20-74 years with assessment of hepatic steatosis were recruited. Exclusion criteria included: (1) ungradable images of hepatic steatosis $(N=$ 941); (2) participants without important covariates: body mass index (BMI), systolic blood pressure (SBP), diastolic blood pressure (DBP), waist circumference, total cholesterol (TC), total triglyceride (TG), high-density lipoprotein cholesterol (HDL), fasting plasma glucose (FPG), fasting insulin and glycosylated hemoglobin (HbA1c) $(N=1,366)$; (3) participants with missing follow-up data $(N=10)$. After exclusion, 12,480 eligible participants were followed up for a medium of 22.8 years (interquartile range 20.7-24.7 years, Figure 1).

\section{Laboratory Measurement and Index Calculation}

Serum biochemistries were measured by the Hitachi 737 automated multichannel chemistry analyzer (Boehringer Mannheim Diagnostics, Inc., Indianapolis, Indiana). Homeostatic Model Assessment for Insulin Resistance (HOMAIR) was adopted to estimate the level of $\beta$-cell function. Methods for non-invasive fibrosis assessment, such as NAFLD fibrosis score (NFS score), AST-to-platelet ratio index (APRI), and fibrosis-4 index (FIB-4), were evaluated by original formulas (Supplementary Table 1).

\section{Definitions and Subgroups}

Categorized assessment of hepatic steatosis by ultrasound encompassed none, mild, moderate and severe, and only mild to severe hepatic steatosis was regarded as evidence of hepatic steatosis (19). NAFLD was diagnosed if an adult with steatosis confirmed by ultrasound without (1) high alcoholic consumption (over one drink daily among women or over two drinks daily among men); (2) presence of hepatitis B surface antigens or antibodies to hepatitis C; (3) iron overload (transferrin saturation $\geq 50 \%$ along with serum ferritin $\geq 400 \mu \mathrm{g} / \mathrm{L}$ in women and $\geq 500$ $\mu \mathrm{g} / \mathrm{L}$ in men) (20). MAFLD was defined by the international expert consensus statement in 2020 (12), including ultrasound confirmed hepatic steatosis plus one of the three criteria: overweight or obesity defined as BMI $\geq 25 \mathrm{~kg} / \mathrm{m}^{2}$, presence of type 2 diabetes mellitus, and metabolic disorders described by any two indicators: (1) waist circumference (WC) $\geq 102 \mathrm{~cm}$ in men or $\geq 88 \mathrm{~cm}$ in women; (2) blood pressure $\geq 140 / 90 \mathrm{mmHg}$ or taking anti-hypertension drugs; (3) raised triglycerides (plasma 
$\mathbf{N}=\mathbf{1 4 , 7 9 7}$ participants in NHANE III ages 20-74 years with results of hepatic/gallbladder ultrasound

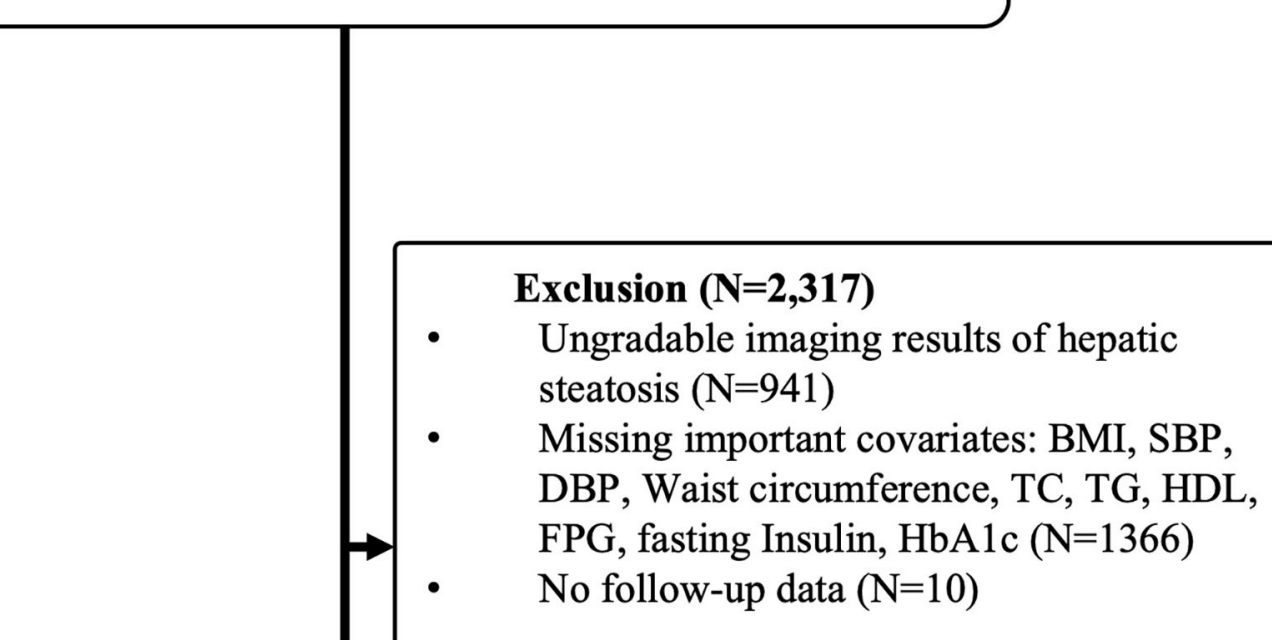

\section{$\mathbf{N}=\mathbf{1 2 , 4 8 0}$ for final analysis}

FIGURE 1 | Flow-chart of the Study. NHANE III, The National Health and Nutrition Examination Survey III (1988-1994). BMI, body mass index; SBP, systolic blood pressure; DBP, diastolic blood pressure; TC, total cholesterol; TG, triglyceride; HDL, high-density lipoprotein cholesterol; FPG, fasting plasma glucose; HbA1c, glycosylated hemoglobin.

triglycerides $\geq 1.70 \mathrm{mmol} / \mathrm{L}$ or taking specific anti-lipid agents); (4) reduced HDL cholesterol (plasma HDL $<1.0 \mathrm{mmol} / \mathrm{L}$ for men and $<1.3 \mathrm{mmol} / \mathrm{L}$ for women or taking specific agents); (5) prediabetes status (FPG 5.6-6.9 mmol/L, or 2-h post-load glucose levels 7.8-11.0 mmol or HbA1c 5.7-6.4\%); (6) HOMA-IR $\geq 2.5$; (7) plasma high-sensitivity C-reactive protein (CRP) level $>2 \mathrm{mg} / \mathrm{L}$.

The presence of diabetes mellitus was defined as a selfreport history of diabetes mellitus, fasting glucose levels (FPG) $\geq 7.0 \mathrm{mmol} / \mathrm{L}, 2$-h post-load glucose levels $\geq 11.0 \mathrm{mmol}$ (for 
participants given an oral glucose tolerance test), $\mathrm{HbAlc} \geq$ $6.5 \%$ or taking diabetes drugs. Hypertension was defined as $\mathrm{BP} \geq 140 / 90 \mathrm{mmHg}$, or taking anti-hypertension drugs. The definition of metabolic syndrome was according to the joint interim statement in 2009 (21). Waist circumference criteria in ATP III ( $\geq 102 \mathrm{~cm}$ in male; $\geq 88 \mathrm{~cm}$ in female) was used for abdominal obesity in the United States. Elevated liver enzymes were defined as AST $>37 \mathrm{U} / \mathrm{L}$ in men and $>31 \mathrm{U} / \mathrm{L}$ in women or $\mathrm{ALT}>40 \mathrm{U} / \mathrm{L}$ in men and $>31 \mathrm{U} / \mathrm{L}$ in women.

According to the NCHS, all-cause death recorded all deceased participants. Main causes of death following the guidelines of International Statistical Classification of Diseases, Injuries, and Causes of Death (ICD-9 before 1998 and ICD10 afterwards) presented as cause-specific mortality (22), consisting of cardiovascular mortality recorded by heart and cerebrovascular diseases, neoplasm mortality recorded by malignant neoplasms in all systems, and diabetes-related mortality recorded by diabetes mellitus.

We further separated the cohort into the four mutually exclusive groups based on definitions of MAFLD and NAFLD. Group $\mathrm{M}+\mathrm{N}$ : participants meet the diagnostic criteria for both MAFLD and NAFLD definitions were in group $\mathrm{M}+\mathrm{N}$; Group N: participants can be defined as NAFLD but not MAFLD; Group M: participants defined as MAFLD but not NAFLD in group $\mathrm{N}$ or $\mathrm{M}$; participants excluded by both definitions were viewed as control group.

\section{Statistical Analysis}

All analysis was weighted by sample weights to reflect population-based estimates. Continuous data were presented as mean and 95\% confidence intervals (geometric mean for variables without normal distribution). Categorical variables were displayed as percentages. The baseline characteristics of the participants among groups were compared by one-way ANOVA test when appropriate for continuous variables or chisq test for categorical variables.

For survival analysis, we used Kaplan-Meier methods to estimate cumulative hazard. To establish cox regression models, the following confounders were considered initially:

- Sociodemographic features: age, sex, race-ethnicity, smoking status.

- Hepatic assessment: alanine transaminase (ALT), aspartate transaminase (AST), CRP, alkaline phosphatase (ALP), FIB-4 score, NFS score, APRI.

- Metabolic assessment: BMI, WC, SBP, DBP, FPG, fasting insulin, HOMAIR, TC, TG.

LASSO regression with minimum mean 10 -fold crossvalidated error was applied for confounder selection to avoid multicollinearity. Among confounders above, we excluded the variables that were penalized to zero by LASSO model (Supplementary Table 2). The LASSO model suggested waist circumference and $\mathrm{HbA1c}$, were stronger indicators than BMI and diabetes, so we used the former instead. Variables with no-zero parameter were classified as above and adjusted stepwise in cox regression models to estimate hazard ratios (HR) and
95\% confidence intervals (95\% CI) for overall and cause-specific mortality. Participants missing relevant covariates were excluded.

Finally, we assessed the association of MAFLD with all-cause, cardiovascular, and neoplasm mortality within subgroups by age (20-39 years, 40-55 years, >55 years), sex, race-ethnicity, diabetes, hypertension, dyslipidemia (raised triglycerides or reduced HDL cholesterol), metabolic syndrome, BMI (Underweight/normal weight: $<25 \mathrm{~kg} / \mathrm{m}^{2}$, overweight: $25-30$ $\mathrm{kg} / \mathrm{m}^{2}$ and obesity: $\left.>30 \mathrm{~kg} / \mathrm{m}^{2}\right)$, severity of hepatic steatosis, NFS, APRI and FIB- 4 score ( $\leq$ weighted mean value, $>$ weighted mean value), presence of other etiologies (alcohol, hepatitis virus, and iron overload), adjusting by age, sex, and race-ethnicity if appropriate. Bonferroni correction was applied and significance was defined as $p<0.0033$.

All statistical analyses were conducted in $\mathrm{R}$ software version 4.0.2. The LASSO regression model was conducted by the Rpackage "glmnet" (23).

\section{RESULTS}

\section{Baseline Characteristics of the Participants With MAFLD and NAFLD}

Of 12,480 participants, 3,909 were diagnosed with MAFLD (weighted prevalence $27.4 \%$ ) and 3,779 were diagnosed with NAFLD (weighted prevalence 27.9\%) (Table 1). Correlation analysis suggested MAFLD was highly concordant with NAFLD (weighted Cohen's kappa coefficient 0.76).

$22.8 \%$ of participants were diagnosed with both NAFLD and MAFLD $(\mathrm{M}+\mathrm{N})$, and the weighted prevalence of only NAFLD (N) and only MAFLD (M) was 5.1 and $4.6 \%$ (Table 1). At baseline, group $\mathrm{N}$ were youngest (mean age: 34.2 years) and complicated with fewest metabolic disorders and histories of cardiovascular diseases. Among these four groups, group $\mathrm{M}$ had the highest proportion of men (66.7\%), ever smokers (70.5\%), the highest prevalence of high alcohol consumption (75.4\%), viral hepatitis (17.3\%), iron overload (10.9\%), hypertension (57.2\%), severe hepatic steatosis (23.8\%) and the highest level of blood pressure (mean SBP: $129 \mathrm{mmHg}$; mean DBP:79.2 mmHg), liver enzymes (mean AST: $33.3 \mathrm{U} / \mathrm{L}$; mean ALT: $31.0 \mathrm{U} / \mathrm{L}$; mean GGT: 69.1 U/L; mean ALP: 88.7 U/L), and fibrosis scores (mean NFS score: -1.45 ; mean APRI score: 0.47; mean FIB-4 score: 1.46). Group $\mathrm{M}+\mathrm{N}$ had the highest prevalence of metabolic syndrome (58.2\%), with highest level of blood lipid (mean TG: $1.89 \mathrm{mmol} / \mathrm{L}$, mean TC: $5.42 \mathrm{mmol} / \mathrm{L}$ ), blood glucose (mean HbA1c: 5.66\%).

\section{Associations of MAFLD/NAFLD With Mortality}

We used LASSO regularization to preselect 11 covariates (Supplementary Table 2), of which age, sex, and race-ethnicity, hepatic assessment (FIB-4 score, NFS score, ALP, and CRP), metabolic parameters (WC, SBP, HbA1c, fasting insulin, TG) were selected for further adjustment. In univariable models, MAFLD increased the risk for all-cause mortality by one-fold compared with non-MAFLD participants. In reference to nonMAFLD participants, MAFLD enhanced the risk for all-cause mortality significantly when age, sex, race-ethnicity, FIB-4, NFS 
TABLE 1 | The characteristics of the participants $(N=12,480)^{*}$.

\begin{tabular}{|c|c|c|c|c|c|c|c|c|c|}
\hline & \multirow[t]{2}{*}{ Overall } & \multicolumn{2}{|c|}{ MAFLD vs. control } & \multicolumn{2}{|c|}{ NAFLD vs. control } & \multicolumn{4}{|c|}{ Separate groups } \\
\hline & & Non-MAFLD & MAFLD & Non-NAFLD & NAFLD & Control & $\mathbf{N}$ & $\mathbf{M}$ & $\mathbf{M}+\mathbf{N}$ \\
\hline$N(\%)$ & $12,480(100)$ & $8,571(72.6)$ & 3,909 (27.4) & $8,701(72.1)$ & 3,779 (27.9) & $8,043(67.5)$ & $528(5.1)$ & $658(4.6)$ & 3,251 (22.8) \\
\hline Age (years) & $42.1(41.8,42.4)$ & $40.1(39.8,40.4)$ & $47.4(46.9,47.9)$ & $40.9(40.6,41.3)$ & $45.1(44.6,45.6)$ & $40.5(40.2,40.9)$ & $34.2(33.2,35.1)$ & $46.9(45.8,47.9)$ & $47.5(47.0,48.0)$ \\
\hline Men (\%) & $5,865(48.7)$ & 3,897 (46.2) & $1,968(55.1)$ & 4,164 (48.4) & $1,701(49.3)$ & 3,702 (47.1) & $195(33.7)$ & $462(66.7)$ & $1,506(52.8)$ \\
\hline \multicolumn{10}{|l|}{ Race-ethnicity (\%) } \\
\hline Non-Hispanic White & 4,648 (76.0) & $3,276(76.7)$ & $1,372(74.2)$ & $3,284(76.4)$ & $1,364(75.1)$ & $3,070(76.6)$ & 206 (78.5) & 214 (73.5) & $1,158(74.4)$ \\
\hline Non-Hispanic Black & $3,544(10.5)$ & 2,658 (11.0) & $886(9.2)$ & $2,668(11.1)$ & $876(9.1)$ & 2,495 (11.1) & $163(9.8)$ & $173(10.5)$ & $713(8.9)$ \\
\hline Mexican-American & $3,765(5.5)$ & $2,272(4.8)$ & $1,493(7.4)$ & $2,371(4.9)$ & 1,394 (7.0) & $2,127(4.8)$ & $145(4.8)$ & $244(7.3)$ & $1,249(7.5)$ \\
\hline Others & $523(8.0)$ & $365(7.5)$ & $158(9.1)$ & $372(7.6)$ & $151(8.9)$ & $351(7.5)$ & $14(6.9)$ & $27(8.7)$ & $131(9.3)$ \\
\hline Ever smoking (\%) & $6,408(55.3)$ & $4,326(54.1)$ & 2,082 (58.3) & $4,554(55.8)$ & $1,854(53.8)$ & $4,101(54.8)$ & $225(44.4)$ & $453(70.5)$ & $1,629(55.9)$ \\
\hline $\mathrm{BMl}, \mathrm{kg} / \mathrm{m}^{2}$ & $26.5(26.4,26.6)$ & $25.0(25.0,25.1)$ & $30.5(30.3,30.7)$ & $25.6(25.5,25.7)$ & $28.9(28.7,29.1)$ & $25.3(25.2,25.4)$ & $21.4(21.2,21.6)$ & $29.9(29.5,30.4)$ & $30.6(30.4,30.8)$ \\
\hline Waist circumference (M), cm & $95.0(94.7,95.4)$ & $91.1(90.7,91.4)$ & $104(103,104)$ & $92.6(92.2,92.9)$ & $101(101,102)$ & $91.6(91.3,92.0)$ & $81.0(79.9,82.2)$ & $102(101,104)$ & $104(104,105)$ \\
\hline Waist circumference (F), cm & $91.7(91.4,92.0)$ & $84.5(84.1,84.8)$ & $101(101,102)$ & $86.2(85.8,86.6)$ & $94.7(93.9,95.5)$ & $85.5(85.1,85.8)$ & $73.9(73.3,74.6)$ & $102(100,105)$ & $101(101,102)$ \\
\hline $\mathrm{SBP}, \mathrm{mmHg}$ & $121(120,121)$ & $118(118,118)$ & $127(127,128)$ & $119(119,120)$ & $124(123,124)$ & $119(118,119)$ & $109(108,110)$ & $129(128,130)$ & $127(127,128)$ \\
\hline DBP, mmHg & $74.3(74.1,74.4)$ & $72.9(72.7,73.1)$ & $77.9(77.6,78.3)$ & $73.6(73.4,73.8)$ & $76.1(75.7,76.4)$ & $73.2(73.0,73.4)$ & $68.9(68.2,69.6)$ & $79.2(78.4,80.1)$ & $77.7(77.3,78)$ \\
\hline $\mathrm{HbA1C}, \%$ & $5.32(5.31,5.34)$ & $5.20(5.19,5.22)$ & $5.64(5.60,5.67)$ & $5.24(5.22,5.25)$ & $5.54(5.51,5.58)$ & $5.22(5.20,5.23)$ & $5.02(4.99,5.05)$ & $5.52(5.43,5.62)$ & $5.66(5.62,5.70)$ \\
\hline HOMA-IR & $2.04(2.02,2.07)$ & $1.70(1.68,1.72)$ & $3.32(3.25,3.4)$ & $1.81(1.78,1.83)$ & $2.81(2.74,2.88)$ & $1.73(1.71,1.75)$ & $1.32(1.28,1.37)$ & $3.35(3.15,3.55)$ & $3.32(3.24,3.41)$ \\
\hline $\mathrm{TG}, \mathrm{mmol} / \mathrm{L}$ & $1.31(1.30,1.32)$ & $1.14(1.13,1.16)$ & $1.87(1.84,1.91)$ & $1.2(1.19,1.21)$ & $1.64(1.61,1.67)$ & $1.17(1.15,1.18)$ & $0.87(0.85,0.90)$ & $1.79(1.71,1.87)$ & $1.89(1.85,1.93)$ \\
\hline $\mathrm{TC}, \mathrm{mmol} / \mathrm{L}$ & $5.13(5.11,5.15)$ & $5.03(5.01,5.05)$ & $5.41(5.38,5.45)$ & $5.09(5.07,5.11)$ & $5.24(5.21,5.28)$ & $5.07(5.05,5.1)$ & $4.52(4.45,4.59)$ & $5.37(5.27,5.46)$ & $5.42(5.38,5.45)$ \\
\hline $\mathrm{HDL}(\mathrm{M}), \mathrm{mmol} / \mathrm{L}$ & $1.13(1.13,1.14)$ & $1.19(1.18,1.20)$ & $1.02(1.01,1.04)$ & $1.18(1.17,1.19)$ & $1.03(1.01,1.04)$ & $1.18(1.17,1.19)$ & $1.29(1.24,1.33)$ & $1.14(1.11,1.18)$ & $1.00(0.98,1.01)$ \\
\hline $\mathrm{HDL}(\mathrm{F}), \mathrm{mmol} / \mathrm{L}$ & $1.38(1.37,1.38)$ & $1.43(1.42,1.44)$ & $1.22(1.21,1.24)$ & $1.42(1.41,1.43)$ & $1.27(1.25,1.28)$ & $1.42(1.41,1.43)$ & $1.49(1.46,1.53)$ & $1.35(1.29,1.4)$ & $1.20(1.19,1.22)$ \\
\hline AST, U/L\# & $21.4(21.2,21.6)$ & $20.2(20.0,20.4)$ & $24.5(24.0,25.1)$ & $21.1(20.8,21.4)$ & $22.2(21.9,22.6)$ & $20.3(20.1,20.5)$ & $19.9(18.8,20.9)$ & $33.3(31.1,35.4)$ & $22.8(22.4,23.2)$ \\
\hline$A L T, U / L^{\#}$ & $18.0(17.8,18.3)$ & $15.9(15.7,16.1)$ & $23.6(23.0,24.2)$ & $16.9(16.7,17.2)$ & $20.8(20.3,21.3)$ & $16.0(15.7,16.2)$ & $14.9(13.9,15.8)$ & $31.0(28.9,33.0)$ & $22.1(21.6,22.7)$ \\
\hline GGT, U/L\# & $29.4(28.6,30.1)$ & $24.7(24.1,25.4)$ & $41.6(39.8,43.4)$ & $28.0(27.1,28.9)$ & $32.8(31.6,34.0)$ & $25.2(24.5,25.9)$ & $18.4(16.1,20.8)$ & $69.1(61.1,77.1)$ & $36.2(34.8,37.6)$ \\
\hline$A L P, U / L^{\#}$ & $80.9(80.4,81.4)$ & $78.3(77.7,78.9)$ & $87.6(86.7,88.6)$ & $79.3(78.7,79.9)$ & $84.8(83.9,85.7)$ & $78.7(78.1,79.3)$ & $72.9(70.7,75.2)$ & $88.7(85.9,91.4)$ & $87.4(86.5,88.4)$ \\
\hline CRP, mg/L & $3.93(3.83,4.03)$ & $3.59(3.47,3.70)$ & $4.83(4.64,5.02)$ & $3.75(3.63,3.87)$ & $4.38(4.21,4.56)$ & $3.69(3.56,3.81)$ & $2.29(2.18,2.41)$ & $4.73(4.22,5.25)$ & $4.85(4.65,5.06)$ \\
\hline NFS score ${ }^{\#}$ & $\begin{array}{c}-2.23(-2.26 \\
-2.21)\end{array}$ & $\begin{array}{c}-2.44(-2.47 \\
-2.41)\end{array}$ & $\begin{array}{c}-1.68(-1.73 \\
-1.63)\end{array}$ & $\begin{array}{c}-2.34(-2.37 \\
-2.30)\end{array}$ & $\begin{array}{c}-1.97(-2.02 \\
-1.92)\end{array}$ & $\begin{array}{c}-2.40(-2.43 \\
-2.36)\end{array}$ & $\begin{array}{c}-3.03(-3.12 \\
-2.93)\end{array}$ & $\begin{array}{c}-1.45(-1.58 \\
-1.32)\end{array}$ & $\begin{array}{c}-1.73(-1.78 \\
-1.68)\end{array}$ \\
\hline APRI score ${ }^{\#}$ & $0.22(0.22,0.22)$ & $0.20(0.20,0.21)$ & $0.26(0.25,0.28)$ & $0.22(0.21,0.23)$ & $0.22(0.21,0.23)$ & $0.20(0.20,0.21)$ & $0.21(0.19,0.23)$ & $0.47(0.39,0.54)$ & $0.22(0.22,0.23)$ \\
\hline FIB-4 score ${ }^{\#}$ & $0.91(0.89,0.92)$ & $0.86(0.85,0.87)$ & $1.03(0.99,1.06)$ & $0.90(0.89,0.92)$ & $0.91(0.89,0.93)$ & $0.87(0.85,0.88)$ & $0.77(0.72,0.81)$ & $1.46(1.27,1.65)$ & $0.94(0.92,0.96)$ \\
\hline \multicolumn{10}{|c|}{ Severity of hepatic steatosis (\%) } \\
\hline None & $7,940(66.5)$ & $7,940(91.7)$ & $0(0)$ & 7,940 (92.3) & $0(0)$ & $7,940(98.6)$ & $0(0)$ & $0(0)$ & $0(0)$ \\
\hline Mild & $1,695(13.5)$ & $387(5.4)$ & $1,308(35.0)$ & $270(2.8)$ & $1,425(41.0)$ & $62(0.8)$ & $325(66.5)$ & $208(33.2)$ & $1,100(35.4)$ \\
\hline Moderate & $1,931(13.6)$ & $204(2.4)$ & $1,727(43.0)$ & 328 (3.3) & $1,603(40.1)$ & $30(0.6)$ & $174(27.4)$ & $298(43.0)$ & $1,429(43.0)$ \\
\hline Severe & $914(6.4)$ & $40(0.5)$ & 874 (22.0) & $163(1.6)$ & $751(18.8)$ & $11(0.1)$ & $29(6.1)$ & $152(23.8)$ & $722(21.6)$ \\
\hline Diabetes (\%) & 1,852 (10.3) & $767(6.0)$ & $1,085(21.6)$ & 957 (7.6) & 895 (17.3) & 767 (6.5) & $0(0)$ & $190(24.0)$ & $895(21.1)$ \\
\hline
\end{tabular}




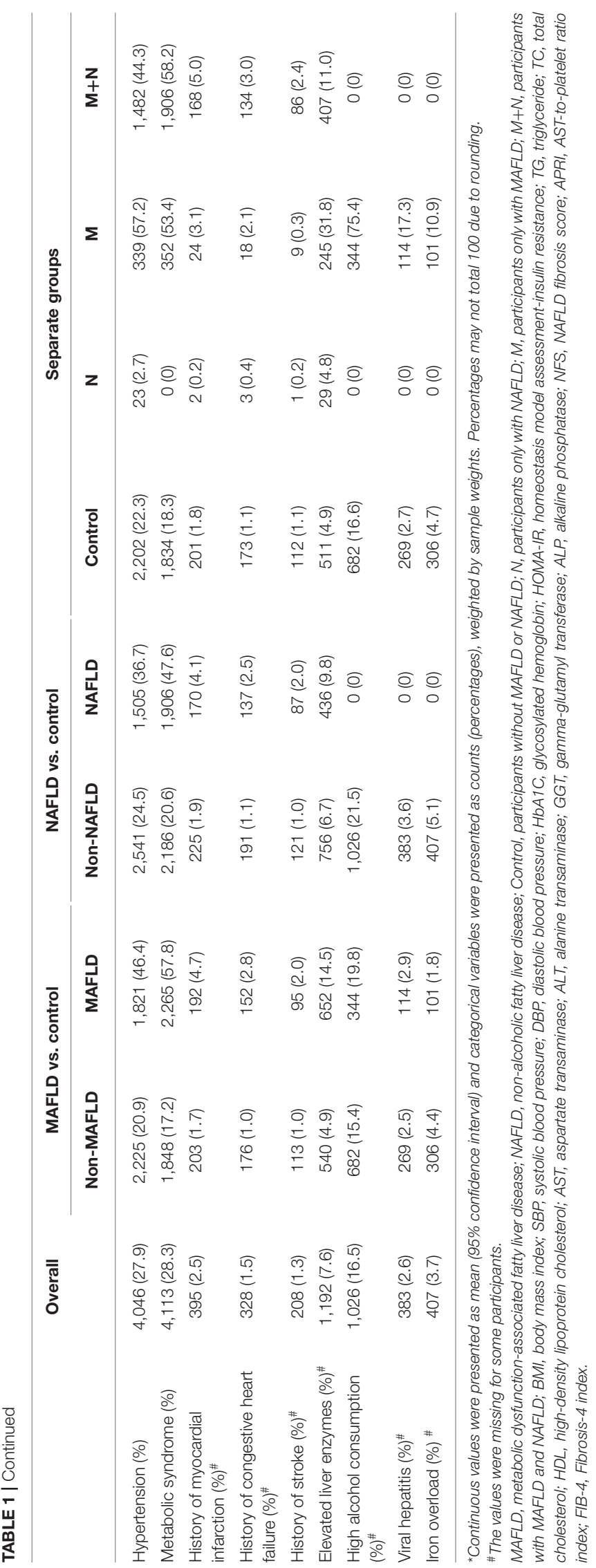

score, ALP, and CRP was adjusted [HR $1.21(1.09,1.33)]$, but this increase was non-significant when waist circumference, HbA1c, SBP, TG, and fasting insulin were further adjusted [HR 1.03 $(0.93,1.15)]$. In reference to non-NAFLD participants, NAFLD increased the risk for all-cause mortality by around 50\%, and the significance was lost after age, sex and ethnicity related factors were corrected [HR 1.05 (0.87, 1.28)] (Table 2). Both MAFLD and NAFLD showed a relatively significant positive correlation with cardiovascular and neoplasm mortality, however, risks of these mortalities were equal between participants with and without MAFLD or NAFLD after age and sex were adjusted. The relative risk of diabetes-related mortality was markedly elevated in participants with either MAFLD or NAFLD even after all factors were adjusted.

We further divided the participants into four groups. In reference to the group without MAFLD and NAFLD (control group), group $\mathrm{N}$ reduced all-cause mortality by around $50 \%$, and the association was non-significant after age, sex and race-ethnicity were adjusted; group $\mathrm{M}$ independently increased the risk of all-cause mortality by $47 \%$; group $\mathrm{M}+\mathrm{N}$ was significantly associated with elevated all-cause mortality unless waist circumference, $\mathrm{HbA1c}$, SBP, TG and fasting insulin were adjusted [HR $0.96(0.86,1.07)$ ] (Figure 2A, Table 2). For cardiovascular mortality, group $\mathrm{M}$ and group $\mathrm{M}+\mathrm{N}$ both showed an increased risk than control in-crude, but this risk was unaltered in group $\mathrm{M}$ after FIB-4, NFS score, CRP and ALP score were adjusted and in group $\mathrm{N}+\mathrm{M}$ after sex and age were adjusted (Figure 2B, Table 2). Group $\mathrm{M}$ independently increased the risk of neoplasm mortality after all confounders were adjusted. The risk of neoplasm mortality was reduced in group $\mathrm{N}$ and enhanced in group $\mathrm{M}+\mathrm{N}$ in reference to control group incrude (Figure 2C, Table 2). Group $\mathrm{M}$ and group $\mathrm{M}+\mathrm{N}$ relatively enhanced risk of diabetes-related mortality unless corrected by metabolic factors, compared with the control group (Figure 2D, Table 2). The risk of group $\mathrm{N}$ in diabetes-related risk was unavailable without enough events.

\section{Subgroup Analysis of MAFLD}

The risk of the MAFLD for overall mortality was similar in subgroups with different age, BMI, severity of hepatic steatosis, diabetes, hypertension, dyslipidemia, metabolic syndrome, FIB4, and other etiologies (Figure 3). Significant heterogeneity was only found in different ethnicities and presence of viral hepatitis (Bonferroni corrected). MAFLD increased risk for allcause mortality in non-Hispanic white race [HR 1.37 (1.22, $1.54)]$, with viral hepatitis [HR $2.56(1.56,4.21)]$ or without viral hepatitis [HR $1.24(1.13,1.37)]$. There was no difference in subgroups in cardiovascular and neoplasm mortality risk in MAFLD (Supplementary Figures 1, 2).

\section{DISCUSSION}

Compared with patients with NAFLD, patients with MAFLD had increased risk for all-cause mortality in a greater magnitude in spite of similar cardiovascular, neoplasm and diabetes-related mortality risk. The nomenclature changes excluded participants who were negatively associated with mortality and captured 
TABLE 2 | Cox regression model for overall and disease-specific mortality of participants.

\begin{tabular}{|c|c|c|c|c|c|}
\hline & Deaths & Unadjusted HR & Model 1 & Model 2 & Model 3 \\
\hline \multicolumn{6}{|c|}{ Overall mortality } \\
\hline MAFLD & 1,561 & $2.07(1.86,2.29)^{\star}$ & $1.27(1.16,1.41)^{\star}$ & $1.21(1.09,1.33)^{\star}$ & $1.03(0.93,1.15)$ \\
\hline NAFLD & 1,326 & $1.47(1.20,1.79)^{\star}$ & $1.05(0.87,1.28)$ & $0.99(0.81,1.20)$ & $0.81(0.66,1.00)$ \\
\hline \multicolumn{6}{|c|}{ Cardiovascular mortality } \\
\hline MAFLD & 409 & $2.01(1.66,2.44)^{\star}$ & $1.17(0.96,1.42)$ & $1.10(0.90,1.34)$ & $0.83(0.68,1.02)$ \\
\hline NAFLD & 352 & $1.53(1.26,1.86)^{\star}$ & $1.07(0.89,1.30)$ & $0.99(0.81,1.21)$ & $0.80(0.65,0.98)^{*}$ \\
\hline \multicolumn{6}{|c|}{ Neoplasm mortality } \\
\hline MAFLD & 356 & $1.78(1.45,2.17)^{\star}$ & $1.16(0.94,1.42)$ & $1.12(0.91,1.39)$ & $1.12(0.88,1.41)$ \\
\hline NAFLD & 307 & $1.31(1.06,1.61)^{\star}$ & $1.01(0.82,1.25)$ & $0.98(0.79,1.22)$ & $0.96(0.76,1.21)$ \\
\hline \multicolumn{6}{|c|}{ Diabetes-related mortality } \\
\hline MAFLD & 99 & $6.86(3.94,11.95)^{\star}$ & $4.57(2.63,7.97)^{\star}$ & $4.40(2.49,7.76)^{\star}$ & $1.84(0.97,3.50)$ \\
\hline NAFLD & 78 & $3.26(1.90,5.59)^{\star}$ & $2.54(1.49,4.34)^{\star}$ & $2.72(1.59,4.63)^{\star}$ & $1.38(0.81,2.37)$ \\
\hline \multicolumn{6}{|c|}{ Overall mortality } \\
\hline Control & 2,139 & Ref & Ref & Ref & Ref \\
\hline $\mathrm{N}$ & 73 & $0.48(0.34,0.68)^{\star}$ & $0.92(0.65,1.31)$ & $0.95(0.65,1.38)$ & $1.09(0.75,1.58)$ \\
\hline M & 308 & $2.76(2.28,3.33)^{\star}$ & $1.87(1.57,2.23)^{\star}$ & $1.73(1.44,2.08)^{\star}$ & $1.47(1.22,1.77)^{\star}$ \\
\hline $\mathrm{N}+\mathrm{M}$ & 1,253 & $1.85(1.65,2.07)^{\star}$ & $1.17(1.05,1.29)^{\star}$ & $1.12(1.00,1.24)^{\star}$ & $0.96(0.86,1.07)$ \\
\hline \multicolumn{6}{|c|}{ Cardiovascular mortality } \\
\hline Control & 551 & Ref & Ref & Ref & Ref \\
\hline N & 15 & $0.46(0.20,1.02)$ & $1.01(0.45,2.30)$ & $0.93(0.36,2.42)$ & $1.24(0.48,3.25)$ \\
\hline M & 72 & $2.35(1.60,3.45)^{\star}$ & $1.53(1.03,2.28)^{\star}$ & $1.47(0.98,2.20)$ & $1.05(0.70,1.58)$ \\
\hline $\mathrm{N}+\mathrm{M}$ & 337 & $1.86(1.51,2.28)^{\star}$ & $1.11(0.91,1.35)$ & $1.03(0.84,1.27)$ & $0.80(0.64,0.98)$ \\
\hline \multicolumn{6}{|c|}{ Neoplasm mortality } \\
\hline Control & 530 & Ref & Ref & Ref & Ref \\
\hline N & 21 & $0.46(0.24,0.89)^{\star}$ & $0.81(0.42,1.56)$ & $0.88(0.46,1.72)$ & $0.89(0.46,1.72)$ \\
\hline M & 71 & $2.16(1.50,3.10)^{\star}$ & $1.54(1.08,2.20)^{\star}$ & $1.59(1.12,2.26)^{\star}$ & $1.58(1.09,2.28)^{\star}$ \\
\hline $\mathrm{N}+\mathrm{M}$ & 285 & $1.63(1.31,2.02)^{\star}$ & $1.08(0.87,1.35)$ & $1.04(0.83,1.31)$ & $1.04(0.81,1.34)$ \\
\hline \multicolumn{6}{|c|}{ Diabetes-related mortality } \\
\hline Control & 66 & Ref & Ref & Ref & Ref \\
\hline N & 0 & NA & NA & NA & NA \\
\hline M & 21 & $9.13(4.15,20.05)^{\star}$ & $6.66(3.03,14.62)^{\star}$ & $5.53(2.61,11.71)^{\star}$ & $2.09(0.71,6.14)$ \\
\hline $\mathrm{N}+\mathrm{M}$ & 78 & $5.86(3.25,10.58)^{\star}$ & $4.01(2.23,7.22)^{\star}$ & $4.02(2.33,6.94)^{\star}$ & $1.78(0.95,3.35)$ \\
\hline
\end{tabular}

${ }^{*} p<0.05$

Values were presented as hazard ratio (95\% confidence interval). Model 1: adjusted by age, sex and race-ethnicity (N = 12,480). Model 2: Model 1+ adjusted by FIB-4 score, NFS score,

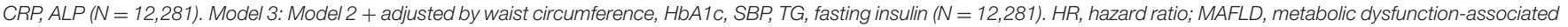

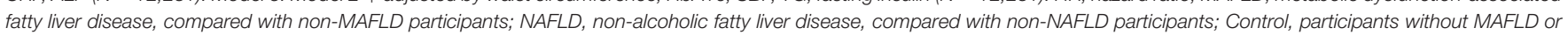
NAFLD; N, participants only with NAFLD; M, participants only with MAFLD; M+N, participants with MAFLD and NAFLD; NA, not applicable.

participants who had higher all-cause mortality risk. The risk of mortality was similar among MAFLD subgroup except for non-Hispanic white race and viral hepatitis comorbidity.

Our study identified that patients diagnosed with new definition would have greater all-cause mortality risk in a medium follow-up time of 22.8 years. The risk for cardiovascular and neoplasm mortality was similar between MAFLD and NAFLD. Similarly, a previous study suggested that the fatal and non-fatal cardiovascular outcomes were similar between NAFLD and MAFLD after a 7-year follow-up (17). This indicated that the term MAFLD emphasized total mortality risk but did not affect the major outcomes of fatty liver. Adjusting confounders for mortality step by step, we found the association between NAFLD and all-cause mortality was non-significant after age and sex were adjusted and the risk of MAFLD on all-cause mortality was largely attributable to the dysregulated metabolic profile. The impact of metabolic disorder on mortality was more prominent in MAFLD compared with NAFLD. The risk of fatty liver on cardiovascular and neoplasm mortality was mainly owning to age, sex and race and our study showed cause-specific mortality was similar between NAFLD and MAFLD.

Some researchers were concerned that this new definition may lose some participants, especially those with severe steatosis (24). However, our study suggested in the patients excluded after the name switch, only $6 \%$ had severe steatosis. The excluded patients were mainly participants with NAFLD without apparent metabolic disorder, who had a "cardio-protective" 


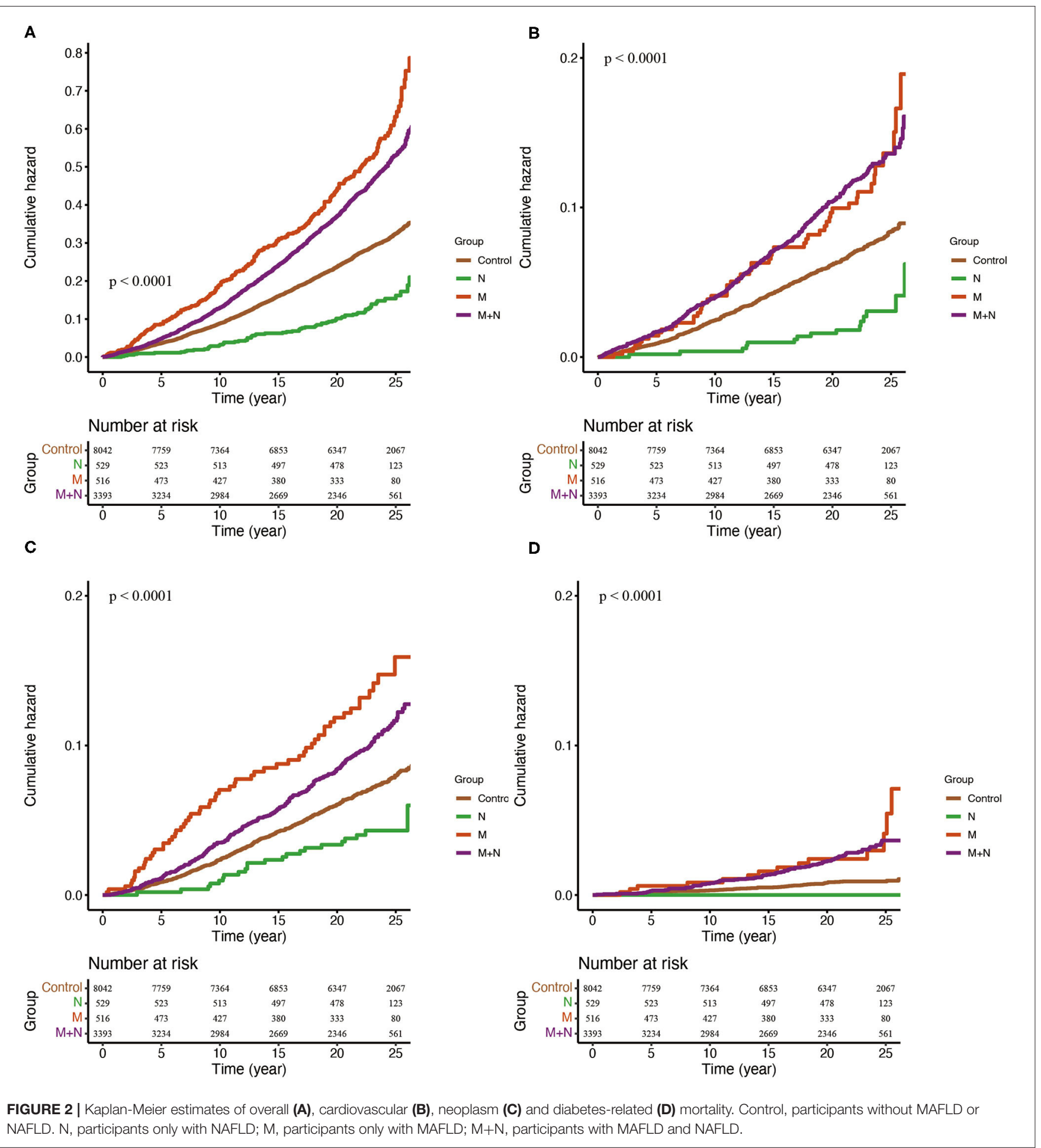

metabolic profile as well as significantly lower liver enzymes and hepatic fibrosis scores. More importantly, they showed reduced risk with mortality possibly owing to their young age and low levels of metabolic disorder. Therefore, the participants excluded might not be the priority for clinical intervention and drug development.
Other researchers found that the change in terminology included more patients with metabolic disorders (14), these patients were included in group $M$ in our study. This group was independently associated with all-cause mortality, especially with neoplasm mortality. They were identified with the worst metabolic profile and advanced 


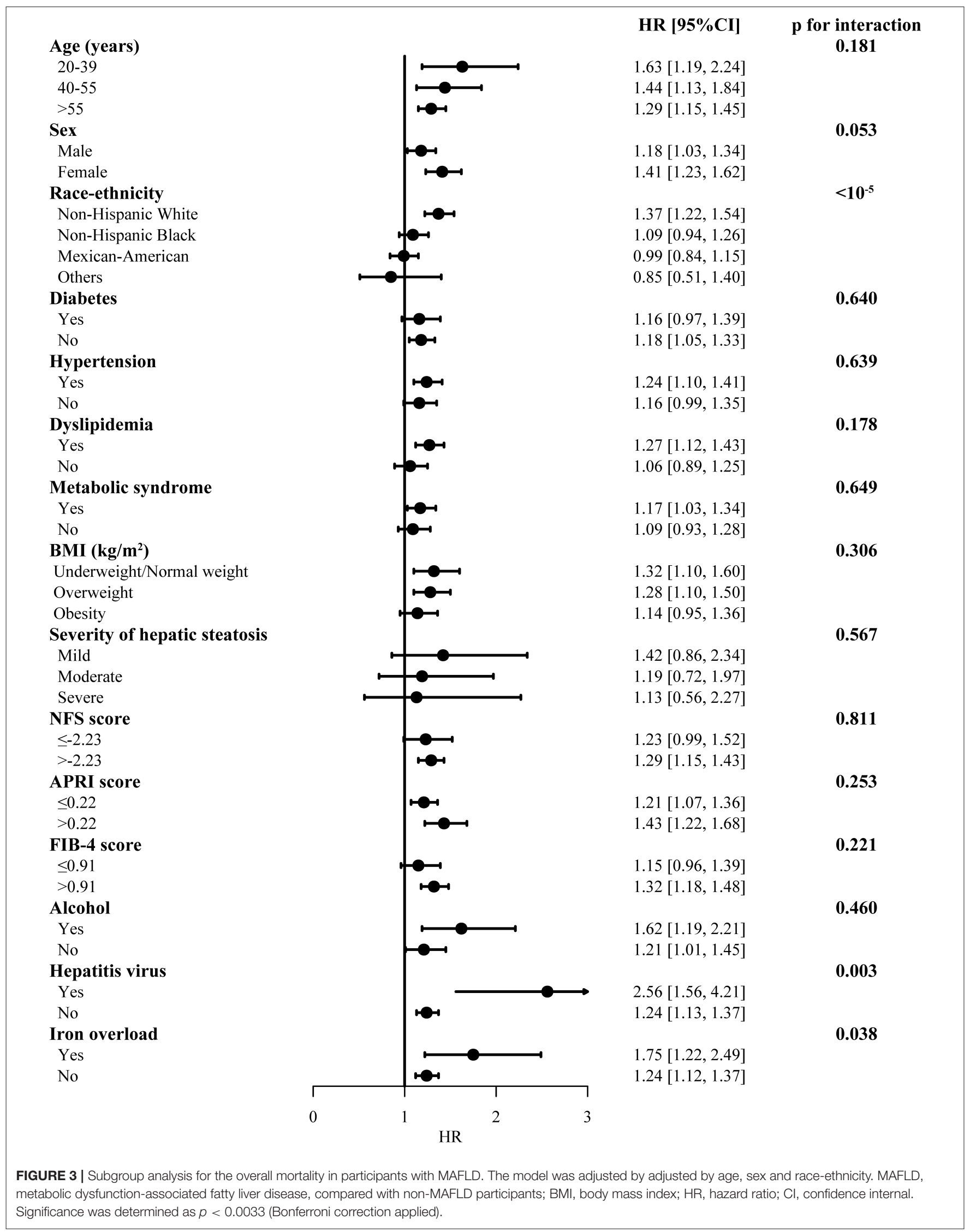


hepatic inflammation and fibrosis, indicating possible worse liver outcomes $(25,26)$. These patients ignored by previous criteria of NAFLD were mainly patients with alcohol abuse, hepatic viral infection and iron overload, which tended to speed up the progression of extracellular and hepatocellular cancer (27). The risk for total mortality and cardiovascular mortality was similar between group $\mathrm{M}$ and group $\mathrm{M}+\mathrm{N}$, indicating the drugs developed for MAFLD may also be applicable for group M. Also, MAFLD with or without other etiology showed no heterogeneity in subgroup analysis regarding all-cause mortality risk. In this scenario, patients in group $\mathrm{M}$ may also benefit from drugs developed for MAFLD.

Similar HRs were observed in subgroups of age, sex, smoking status, metabolic dysfunction, hepatic steatosis, FIB-4, and different etiologies. We only detected significant heterogeneity of race-ethnicity and presence of hepatic viral infection in all-cause mortality, after Bonferroni correction ( $p_{\text {interaction }}<$ $0.05 / 13$ ) was applied. The hazard ratio of MAFLD was highest in non-Hispanic white, followed by non-Hispanic black, Mexican-American and other races. As non-Hispanic whites accounted for over three fourth in the United States, they may be the group most affected by this disease. The design for clinical trials could possibly consider stratify patient recruitment according to ethnicities. We also observed significantly greater risks for overall mortality among patients with MAFLD secondary to hepatic viral infection, whereas failing tested the heterogeneity in cause-specific mortality. As the seventh leading cause of death globally and an increasing epidemic trend (28), hepatic virus infection may primarily accelerate the course of liver-related especially with comorbidity of fatty liver disease. Our results suggested drug development for patients with MAFLD should take racial difference and viral hepatitis infection into consideration in the future.

Our study used a large population-based prospective cohort with long follow-up to analyze the association between MAFLD/NAFLD and mortality. However, there are several limitations to our current study. The liver outcomes, especially fine categorization of liver cancer and advanced cirrhosis, were still needed for a comprehensive vision on the natural history of fatty liver disease. We were unable to perform the analysis due to data acquisition limitations. However, the incidence rate of cardiovascular mortality was around 5 times higher than liver-specific mortality in NAFLD (1). Extrahepatic neoplasm may be a primary source for extra mortality in NAFLD (5). In the NHANES III cohort, NAFLD showed similar liver-related mortality with non-NAFLD controls and liverrelated mortality only account for $<2 \%$ of total mortality (19, 20). Liver-related mortality become more relevant when the stages of steatosis progressed, however, this required precise categorization of fatty liver stages which we were unable to perform. The liver-related outcomes may change when other etiology, e.g., alcoholic liver disease (AFLD) and viral hepatitis, was included in MAFLD. Nevertheless, one study suggested in a fatty liver cohort with mixed background of NAFLD and AFLD, mortality from cardiovascular disease and total neoplasm still surpassed liver cirrhosis (29). In addition, the new definition emphasized the presence of metabolic derangements which mainly leads to elevated cardiovascular risk. By this means, we used total mortality, cardiovascular mortality and cancer mortality as our outcomes should still provide robust information to reveal the impact of nomenclature change. Secondly, hepatic steatosis in adults was detected by imaging techniques instead of liver histology, possibly weakening the reliability of the diagnosis of NAFLD. But one qualified metaanalysis showed high sensitivity and specificity in the detection of moderate-severe hepatic steatosis by ultrasound (30). With the improvement of ultrasound, imaging techniques still had limited sensitivity to detect mild steatosis (31). The study only used ultrasound results 30 years ago and the sensitivity of ultrasound detection was greatly improved in recent years (32). Thirdly, we did not excluded the drug-induced hepatotoxicity in the NAFLD definition since we were unable to establish causal relationship between drug use history and fatty liver in an epidemiological survey. One study reported very small portion of participants taken drugs related to hepatotoxicity, and there was no significant difference in mortality after excluding them (33). Finally, some non-statistically significant findings may be related to the limited sample size especially in subgroup analysis, indicative of lower power of the study. More similar studies should be designed and integrated to reduce type 2 error.

In conclusion, using baseline and follow-up data from the cohort of NHANES III, we found MAFLD had an enhanced risk for mortality and similar risk for cause-specific mortality with NAFLD. The definition MAFLD emphasized the role of metabolic disorder on the outcomes of fatty liver since the risk of MAFLD for mortality was largely attributable to its metabolic disorder. The switch from NAFLD to MAFLD captured participants with higher mortality risk regardless of losing some patients with reduced mortality risk. Ethnic differences and the presence of virus hepatitis should be taken into consideration when trials investigating outcomes for MAFLD were implemented.

\section{DATA AVAILABILITY STATEMENT}

Publicly available datasets were analyzed in this study. This data can be found here: https://www.cdc.gov/nchs/ nhanes/nh3data.htm.

\section{ETHICS STATEMENT}

The studies involving human participants were reviewed and approved by NCHS Ethics Review Board. The patients/participants provided their written informed consent to participate in this study.

\section{AUTHOR CONTRIBUTIONS}

QH, XZo, and LJ designed the research. QH and XZo collected, analyzed the data, and drafted the manuscript. XW and XZh 
revised the manuscript. All authors contributed to the article and approved the submitted version.

\section{FUNDING}

The work was supported by a grant from the National Key R\&D Program of China to LJ (2016YFC1304901) and XZh (2016YFC1305603) and from the Beijing Nova Program of

\section{REFERENCES}

1. Younossi ZM, Koenig AB, Abdelatif D, Fazel Y, Henry L, Wymer M. Global epidemiology of nonalcoholic fatty liver disease-Meta-analytic assessment of prevalence, incidence, and outcomes. Hepatology. (2016) 64:7384. doi: 10.1002/hep.28431

2. Marchesini G, Day CP, Dufour J-F, Canbay A, Nobili V, Ratziu $\mathrm{V}$, et al. EASL-EASD-EASO clinical practice guidelines for the management of non-alcoholic fatty liver disease. J Hepatol. (2016) 64:1388-402. doi: 10.1016/j.jhep.2015.11.004

3. Adams LA, Waters OR, Knuiman MW, Elliott RR, Olynyk JK. NAFLD as a risk factor for the development of diabetes and the metabolic syndrome: an eleven-year follow-up study. Am J Gastroenterol. (2009) 104:8617. doi: 10.1038/ajg.2009.67

4. Bril F, Sninsky JJ, Baca AM, Superko HR, Portillo Sanchez P, Biernacki $\mathrm{D}$, et al. Hepatic steatosis and insulin resistance, but not steatohepatitis, promote atherogenic dyslipidemia in NAFLD. J Clin Endocrinol Metab. (2016) 101:644-52. doi: 10.1210/jc.2015-3111

5. Simon TG, Roelstraete B, Khalili H, Hagström H, Ludvigsson JF. Mortality in biopsy-confirmed nonalcoholic fatty liver disease: results from a nationwide cohort. Gut. (2021) 70:1375-82. doi: 10.1136/gutjnl-2020-3 22786

6. Targher G, Byrne CD, Lonardo A, Zoppini G, Barbui C. Non-alcoholic fatty liver disease and risk of incident cardiovascular disease: a meta-analysis. J Hepatol. (2016) 65:589-600. doi: 10.1016/j.jhep.2016. 05.013

7. Liu Y, Zhong GC, Tan HY, Hao FB, Hu JJ. Nonalcoholic fatty liver disease and mortality from all causes, cardiovascular disease, and cancer: a meta-analysis. Sci Rep. (2019) 9:11124. doi: 10.1038/s41598-019-4 7687-3

8. Ong JP, Pitts A, Younossi ZM. Increased overall mortality and liver-related mortality in non-alcoholic fatty liver disease. J Hepatol. (2008) 49:608612. doi: 10.1016/j.jhep.2008.06.018

9. Younossi Z, Anstee QM, Marietti M, Hardy T, Henry L, Eslam M, et al. Global burden of NAFLD and NASH: trends, predictions, risk factors and prevention. Nat Rev Gastroenterol Hepatol. (2018) 15:11. doi: 10.1038/nrgastro. 2017.109

10. Haflidadottir S, Jonasson JG, Norland H, Einarsdottir SO, Kleiner $\mathrm{DE}$, Lund $\mathrm{SH}$, et al. Long term follow-up and liver-related death rate in patients with non-alcoholic and alcoholic related fatty liver disease. BMC Gastroenterol. (2014) 14:1-10. doi: 10.1186/1471-230X14-166

11. Eslam M, Sanyal AJ, George J, Sanyal A, Neuschwander-Tetri B, Tiribelli C, et al. MAFLD: a consensus-driven proposed nomenclature for metabolic associated fatty liver disease. Gastroenterology. (2020) 158:1999-2014.e1. doi: 10.1053/j.gastro.2019. 11.312

12. Eslam M, Newsome PN, Sarin SK, Anstee QM, Targher G, Romero-Gomez $\mathrm{M}$, et al. A new definition for metabolic dysfunction-associated fatty liver disease: An international expert consensus statement. J Hepatol. (2020) 73:202-9. doi: 10.1016/j.jhep.2020.03.039

13. Targher G. Concordance between MAFLD and NAFLD diagnostic criteria in 'real-world' data. Liver Int. (2020) 40:2879-80. doi: 10.1111/liv. 14623
Science and Technology (Z191100001119026) and National Nature Science Foundation of China (81800515) to XZo.

\section{SUPPLEMENTARY MATERIAL}

The Supplementary Material for this article can be found online at: https://www.frontiersin.org/articles/10.3389/fmed. 2021.693507/full\#supplementary-material

14. Lin S, Huang J, Wang M, Kumar R, Liu Y, Liu S, et al. Comparison of MAFLD and NAFLD diagnostic criteria in real world. Liver Int. (2020) 40:2082-9. doi: 10.1111/liv.14548

15. Ye Q, Zou B, Yeo YH, Li J, Huang DQ, Wu Y, et al. Global prevalence, incidence, and outcomes of non-obese or lean non-alcoholic fatty liver disease: a systematic review and meta-analysis. Lancet Gastroenterol Hepatol. (2020) 5:739-52. doi: 10.1016/S2468-1253(20)30077-7

16. Younossi ZM, Rinella ME, Sanyal A, Harrison SA, Brunt E, Goodman Z, et al. From NAFLD to MAFLD: implications of a premature change in terminology. Hepatology. (2020) 73:1194-8. doi: 10.1002/hep.31420

17. Niriella MA, Ediriweera DS, Kasturiratne A, De Silva ST, Dassanayake AS, De Silva AP, et al. Outcomes of NAFLD and MAFLD: Results from a community-based, prospective cohort study. PLoS One. (2021) 16:e0245762. doi: 10.1371 /journal.pone.0245762

18. Centers for Disease Control and Prevention (CDC). NHANES Questionnaires, Datasets, and Related Documentation. CDC website. Available online at: https://wwwn.cdc.gov/nchs/nhanes/default.aspx (accessed January 13, 2020).

19. Alvarez CS, Graubard BI, Thistle JE, Petrick JL, McGlynn KA. Attributable fractions of nonalcoholic fatty liver disease for mortality in the United States: results from the third national health and nutrition examination survey with 27 years of follow-up. Hepatology. (2020) 72:430-40. doi: 10.1002/hep. 31040

20. Lazo M, Hernaez R, Bonekamp S, Kamel IR, Brancati FL, Guallar E, et al. Non-alcoholic fatty liver disease and mortality among US adults: prospective cohort study. BMJ. (2011) 343:d6891. doi: 10.1136/bmj.d6891

21. Alberti KGMM, Eckel RH, Grundy SM, Zimmet PZ, Cleeman JI, Donato KA, et al. Harmonizing the metabolic syndrome. Circulation. (2009) 120:16405. doi: 10.1161/CIRCULATIONAHA.109.192644

22. National Center for Health Statistics. Office of Analysis and Epidemiology, Public-use Linked Mortality File. (2015). Hyattsville, Maryland. Available online at: https://www.cdc.gov/nchs/data-linkage/mortality-public.htm (accessed January 11, 2021).

23. Tibshirani R. The lasso method for variable selection in the Cox model. Stat Med. (1997) 16:385-95. doi: 10.1002/(SICI)10970258(19970228)16:4<385::AID-SIM380>3.0.CO;2-3

24. Huang J, Kumar R, Wang M, Zhu Y, Lin S. MAFLD criteria overlooks a number of patients with severe steatosis: is it clinically relevant? J Hepatol. (2020) 73:1265-7. doi: 10.1016/j.jhep.2020.06.016

25. Larsson SC, Wolk A. Overweight, obesity and risk of liver cancer: a meta-analysis of cohort studies. Br J Cancer. (2007) 97:1005-8. doi: 10.1038/sj.bjc.6603932

26. Wang P, Kang D, Cao W, Wang Y, Liu Z. Diabetes mellitus and risk of hepatocellular carcinoma: a systematic review and meta-analysis. Diabetes Metab Res Rev. (2012) 28:109-22. doi: 10.1002/dmrr.1291

27. Bagnardi V, Rota M, Botteri E, Tramacere I, Islami F, Fedirko V, et al. Alcohol consumption and site-specific cancer risk: a comprehensive doseresponse meta-analysis. Br J Cancer. (2015) 112:580-93. doi: 10.1038/bjc. 2014.579

28. Stanaway JD, Flaxman AD, Naghavi M, Fitzmaurice C, Vos T, Abubakar I, et al. The global burden of viral hepatitis from 1990 to 2013: findings from the global burden of disease study 2013. Lancet. (2016) 388:10818. doi: 10.1016/S0140-6736(16)30579-7

29. Soderberg C, Stål P, Askling J, Glaumann H, Lindberg G, Marmur J, Hultcrantz R. Decreased survival of subjects with elevated liver function tests 
during a 28-year follow-up. Hepatology. (2010) 51:595-602. doi: 10.1002/hep. 23314

30. Hernaez R, Lazo M, Bonekamp S, Kamel I, Brancati FL, Guallar E, et al. Diagnostic accuracy and reliability of ultrasonography for the detection of fatty liver: a meta-analysis. Hepatology. (2011) 54:1082-90. doi: 10.1002/hep. 24452

31. Saadeh S, Younossi ZM, Remer EM, Gramlich T, Ong JP, Hurley M, et al. The utility of radiological imaging in nonalcoholic fatty liver disease. Gastroenterology. (2002) 123:745-50. doi: 10.1053/gast.2002.35354

32. Nguyen VH, Le MH, Henry L, Cheung R. Response to: comparison of MAFLD and NAFLD diagnostic criteria in real world. Liver Int. (2020) 40:3145. doi: 10.1111/liv.14664

33. Dunn W, Xu R, Wingard DL, Rogers C, Angulo P, Younossi ZM, et al. Suspected nonalcoholic fatty liver disease and mortality risk in a population-based cohort study. Am $J$ Gastroenterol.

(2008)

103:2263. doi: 10.1111/j.1572-0241.2008.02034.x

Conflict of Interest: The authors declare that the research was conducted in the absence of any commercial or financial relationships that could be construed as a potential conflict of interest.

Copyright (c) 2021 Huang, Zou, Wen, Zhou and Ji. This is an open-access article distributed under the terms of the Creative Commons Attribution License (CC BY).

The use, distribution or reproduction in other forums is permitted, provided the original author(s) and the copyright owner(s) are credited and that the original publication in this journal is cited, in accordance with accepted academic practice. No use, distribution or reproduction is permitted which does not comply with these terms. 